\title{
Diachronic Semantic Shift of Sequential Conjunction: the Causal to Conditional Path*
}

\author{
Yurie Hara \\ Hokkaido University
}

\begin{abstract}
This paper analyzes the semantic shift of a Japanese construction V-e-ba from causal to conditional. The conventional meaning of the V-e-ba construction is a sequential conjunction in the sense of update semantics, i.e., $c[\varphi$-e-ba $\psi]=$ $c[\varphi][\psi]$. The causal meaning in Old Japanese is obtained by an I-implicature, while the conditional meaning in Modern Japanese is obtained by Q-implicatures. The proposed diachronic development is in accordance with Deo's (2015) Evolutionary Game Theory model.
\end{abstract}

Keywords: historical linguistics, semantic shift, conjunction, causality, conditional, pragmatics, implicatures, evolutionary game theory

\section{V-e-ba}

This paper analyzes the diachronic semantic shift of the Japanese V-e-ba construction. In Old Japanese (OJ), V-e-ba appears to mark a causal adjunct clause as can be seen in the use of causal connectives, because in the English translation (1a) and node in the Modern Japanese (ModJ) translation (1b).

a. kurushiki koto nomi masar-e-ba, ito itau omohiwabitaru wo harsh things only increase-E-BA, very much depressed ACC 'Because only harsh things increased, Ko'oi was very much depressed.'

(Old Japanese; Genji, 11th C)

b. tsurai koto bakari fueteiku node, Ko'oi-ga taisoo hidoku harsh things only increase because, Ko'oi-NOM very much tohoonikurete iru no $\mathrm{O}$ depressed PROG NML ACC

* I would like to thank anonymous SALT reviewers for their valuable comments. I also appreciate the discussions with Michael Yoshitaka Erlewine, Makoto Kanazawa, Magdalena Kaufmann, Satoshi Kinsui, Tomokazu Koyanagi, Kunihiko Kuroki, Toshinobu Ogiso, Kinuhata Tomohide and participants at Semantics Research Group, TwiFULL SLiM and SALT. The project is supported by JSPS Kiban (C) "Semantic-Pragmatic Interfaces at Left Periphery: a neuroscientific approach" (18K00589; PI Yurie Hara). All remaining errors are mine.

C)2019 Hara 
Diachronic Semantic Shift of Sequential Conjunction

(Modern Japanese)

On the other hand, the V-e-ba form in ModJ appears to mark a conditional adjunct (antecedent) as in (2a), while in OJ the conditional is marked with V-a-ba form as in (2b).

a. uramu no-ga mottomona ten-mo kawairashiku bokashite hate NML-NOM reasonable point-ADD sweetly vaguely i-e-ba, sorenitsukete otoko-no aijoo-mo masu koto deshoo say-E-BA, as.it.goes men-GEN love-ADD increase NML will 'Even the things you definitely hate, if you just mention them sweetly, men will love you more.'

(Modern Japanese)

b. uramu bekaram fushi-o-mo, nikukarazu kasumenas-a-ba, hate should thing-ACC-ADD sweetly mention-A-BA, sorenitsukete ahare-mo masarinu-besi as.it.goes love-add increase-should

(Old Japanese; Genji, 11th C)

Furthermore, in Middle Japanese (MidJ), the use of V-e-ba as logical/symmetric conjunction has emerged as in (3).

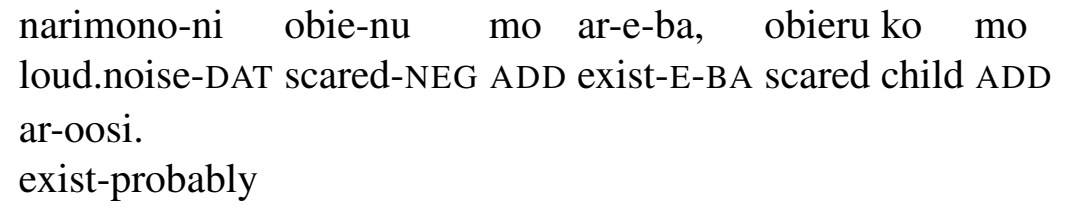

(Middle Japanese; Ukiyoburo, 19th C)

The goal of this paper is to account for how the interpretation of V-e-ba shifted from causal to conditional (via logical/symmetric conjunction). The core semantics of the $\mathrm{V}-e-b a$ construction is a sequential conjunction in the sense of update semantics, i.e., $c[\varphi$-e-ba $\psi]=c[\varphi][\psi]$. The causal meaning in OJ is obtained by an I-implicature (conjunction buttressing), while the conditional meaning in ModJ is obtained by Q-implicatures. The proposed diachronic development can be formalized by Deo's (2015) Evolutionary Game Theory that underpins the grammaticalization paths from the semantic-pragmatic perspective.

\section{Puzzles of the Traditional Grammar}

\subsection{Settled Conditional to Hypothetical Conditional}

In the traditional Japanese grammar (e.g., Sakakura 1958), two verbal morphemes adjacent to - $b a$ in $\mathrm{OJ}$ are said to mark whether the event expressed by the verb is 


\begin{tabular}{l|ll}
\hline & Old Japanese & Modern Japanese \\
\hline $\mathrm{V}-a$ & mizen 'unsettled/irrealis' & mizen 'unsettled/irrealis' \\
$\mathrm{V}-e$ & izen 'settled/realis' & katee 'hypothetical' \\
$\mathrm{V}-a-b a$ & katee jooken 'hypothetical conditional' & N/A \\
$\mathrm{V}-e-b a$ & kakutee jooken & katee jooken \\
& 'settled conditional' & 'hypothetical conditional'
\end{tabular}

Table 1 Terminology Change of Traditional Grammar

settled or not: $-a$ and $-e$ are called mizen 'unsettled/irrealis' and izen 'settled/realis', respectively. Together with the assumption that - $b a$ unambiguously marks conditional, the V-a-ba and V-e-ba constructions are named katee jooken 'hypothetical conditional' and kakutee jooken 'settled conditional', respectively.

Following Sakakura's terminology, Kobayashi (1996) explains the semantic shift of V-e-ba from causal to conditional as follows. First, $\varphi-e-b a \psi$ in OJ expresses conditional dependency between two settled propositions, which gives rise to a causal interpretation, $\varphi$ causes $\psi$. This causal dependency between $\varphi$ and $\psi$ had been generalized over time and the V-e-ba construction has gained a hypothetical interpretation, if $\varphi$, then $\psi$. At the same time, the realis/settledness feature of V-e is lost. Thus, in the traditional grammar, the -e morpheme in ModJ is now called katee kee 'hypothetical form'. This historical change of the terminology is summarized in Table 1.

\subsection{Puzzles}

The traditional account sketched above is puzzling in at least three respects. First, it is unclear what motivates the generalization of the causal $e-b a$ into the conditional. In particular, OJ already had the $a-b a$ conditional which seems to express the genuine conditional. Second, it is mysterious why the settledness/realis feature of $-e$ is lost.

Finally, the traditional explanation is puzzling in view of Traugott \& Dasher's (2002) generalizations of language change: "Meanings tend to become increasingly based in the speaker's subjective belief state/attitude toward the proposition" (p. 95), and "meanings become increasingly more pragmatic and procedural" (p. 40) in that they express meta-linguistic relations between contentful meanings. To illustrate, let us take the English connective because and see how its interpretation has become more subjective and procedural over time. In (4), three types of causation are demonstrated. The causation expressed in (4a) is more or less an objective one between two eventualities, while the one in (4b) involves the speaker's subjective/epistemic 
Diachronic Semantic Shift of Sequential Conjunction

reasoning on why she thinks John loved her. Furthermore, the becasue-clause in (4c) indicates the motivation for why the speaker is performing such a speech act rather than the propositional content of the main clause.

(4) a. John came back because he loved her.

(content)

b. John loved her, because he came back.

(epistemic)

c. What are you doing tonight, because there's a good movie on.

(speech act)

(Sweetser 1990: 77)

Going back to the causal to conditional shift of the V-e-ba construction, a causal statement like (1a) is more subjective and procedural in that it involves the speaker's judgment that there is a causal dependency between two facts, while a conditional statement like (2a) is less subjective and less procedural in that it merely expresses quantification over event predicates. Thus, the claim that V-e-ba shifted from causative to conditional does not fit the general trend of semantic change.

\section{Proposal}

I propose that the semantics of the $\mathrm{V}-e-b a$ construction is a sequential conjunction based on Fukuda's (2006) observation. In Section 3.1, I first review Fukuda's (2006) analysis with some corpus data which support his claim. Section 3.2 presents my own analysis.

\subsection{Fukuda (2006): $e-b a$ as conjunction}

Fukuda (2006) presents convincing evidence against the traditional view and claims that the $b a$ particle is ambiguous: The particle $b a_{1}$ in $\mathrm{V}-e-b a$ is a conditional maker while $b a_{2}$ in V-e-ba is not a marker of conditional but a marker of conjunction. Furthermore, the verbal morphemes $-a$ and $-e$ are not markers of (un)settledness/(ir)realis but markers of syntactic positions. I translate Fukuda's claim in generative terms as follows: $-a$ is a marker of infinite ([-FINITE]) Aspect Phrase (AspP) as depicted in (5), while $-e$ is a marker of finite ([+FINITE]) $\mathrm{CP}$ as in (6).

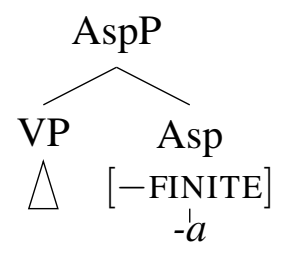




\begin{tabular}{|c|c|c|}
\hline & $-a$ & $-e$ \\
\hline$m-$ & 0 & 1327 \\
\hline ram- & 0 & 187 \\
\hline kem- & 0 & 107 \\
\hline
\end{tabular}

Table 2 Co-occurrences of OJ modalities and verbal morphology in the Corpus of Historical Japanese (CHJ)

(6)

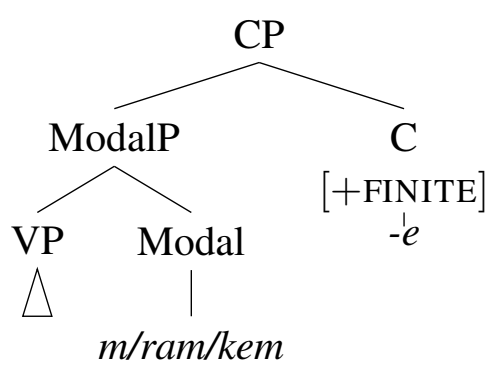

Fukuda (2006) motivates his claim with the following observational fact. As already indicated in the tree structures above, $-a$ cannot embed a modal while $-e$ can. That is, OJ modals of probability, $m$, ram, kem cannot be followed by - $a$ (i.e., ${ }^{*} m-a$, ${ }^{*}$ ram- $\left.a,{ }^{*} k e m-a\right)$, while $m-e$, ram-e, kem-e forms are available. An example of $m-e$ is given in (7).

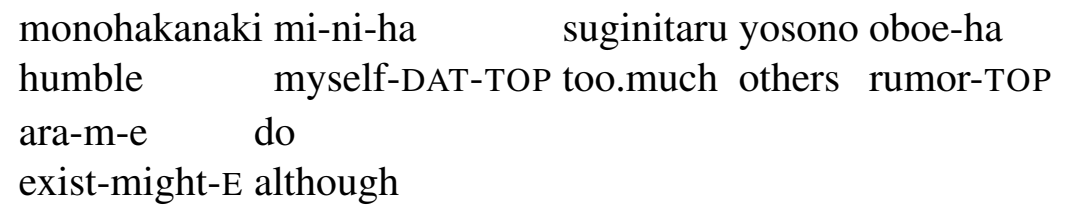

'Although there might be some rumors that it is too much for a humble person like me.'

(Old Japanese; Genji, 11th C)

Furthermore, this distributional pattern can be attested in the Corpus of Historical Japanese $(\mathrm{CHJ}){ }^{1}$ There are zero occurrences of $m$ - $a$, ram- $a$, and kem- $a$ while $m$ - $e$, ram-e, kem-e forms frequently occur.

Therefore, semantically speaking, clauses headed by - $a$ denote event predicates or unsaturated propositions, while clauses headed by $-e$ denote saturated propositions. Thus, (2b) with $\mathrm{V}-a-b a_{1}$ is a genuine conditional which expresses quantification over event predicates (Kratzer 1991), while (1b) with V-e-ba conjunction of two saturated propositions.

1 National Institute for Japanese Language and Linguistics (2019) "Corpus of Historical Japanese" (Version 2019.3, Chunagon Version 2.4.4) https://chunagon.ninjal.ac.jp/ (accessed August 1, 2019). 
Diachronic Semantic Shift of Sequential Conjunction

To summarize, according to Fukuda's (2006) analysis, the clause headed by the - $a$ morpheme is an AspP which denotes an event predicate or an unsaturated proposition, while the clause headed by the $-e$ morpheme is a $\mathrm{CP}$ which denotes a saturated proposition. Furthermore, Fukuda (2006) claims that the particle $b a$ is ambiguous between conditional and conjunction. Thus, $\varphi-a-b a-\psi$ is a genuine conditional while $\varphi-e-b a-\psi$ is a conjunction of two facts.

\subsection{Analysis}

I propose that the default semantics of $\varphi-e-b a-\psi$ is sequential conjunction in update semantics (Stalnaker 1968; Heim 1982):

\section{(8) Proposal 1}

$$
c[\varphi \text {-e-ba } \psi]=c[\varphi][\psi] .
$$

Thus, the semantic interpretation of (1a) is: 'only harsh things increased AND she was very much depressed'. Indeed, (9) shows that OJ $\varphi-e-b a-\psi$ expressed a sequential conjunction of events in chronological order, ' $\varphi$ and then $\psi$ ', rather than a causal relation.

(9) sore-o mir-e-ba, sansun bakari naru hito, ito utsukushiute witar-i. it-ACC see-E-BA 3.inches only COP person very lovely exist-PERF 'He (the old man) looked at it (the bamboo shoot) and then there was a person, who was only three inches tall, sitting very lovely.'

(Old Japanese; Taketori, 9-10th C)

The causal interpretation of $\varphi-e-b a-\psi$ in (1a) arises from pragmatic/Gricean reasoning, i.e., Levinson's (2001) I-implicature/conjunction buttressing.

(10) Proposal 2

The causal meaning of $e-b a$ is an I-implicature.

Levinson (2001) argues that English conjunction and can undergo pragmatic enrichment via Gricean reasoning, in particular I-principle. I-principle allows the addressee to enrich the semantic meaning of the speaker's utterance so that it fits our stereotypical expectations:

(11) The I-principle Speaker: Do not say more than is required.

Addressee: What is generally said is stereotypically and specifically exemplified. (adapted from Huang 2007: 58)

For instance, the semantic interpretation of (12) is just a conjunction of two events, but the addressee will try to causally connect the two events described: 
(12) John turned the key and the engine started.

I-implicates

John turned the key, therefore the engine started.

Similarly, in (1a), repeated here as (13), the use of conjunction $e$-ba I-implicates the causal interpretation as a result of the pragmatic enrichment:

kurushiki koto nomi masar-e-ba, ito itau omohiwabitaru wo.

a. semantic interpretation:

'Only harsh things increased and she was very much depressed'.

b. I-implicature:

'Only harsh things increased, therefore she was very much depressed.'

Finally, how did the conditional interpretation of $e-b a$ in ModJ emerge? My answer is that the conditional meaning is obtained via Q-implicatures:

(14) Proposal 3

The conditional meaning of $e$-ba obtains from Q-implicatures.

First, note that if $c$, the input context to be updated by $\varphi-e-b a-\psi$, is a suppositional context rather than the utterance context, we obtain the ModJ-style conditional interpretation, $\varphi \rightarrow \psi$ (Roberts 1996; Kaufmann 2000).

Furthermore, along the diachronic development, morphemes marked specifically for causal and symmetric conjunction have emerged. As for causality, kara 'because' and node 'because' emerged in 17th C and in 19th C, respectively (Kobayashi 1996). Figure 1 shows the diachronic distribution of the constructions that mark causality. As can be seen, in the Middle Era (12-17th C), causality was expressed by $e$-ba for more than $60 \%$ of the time while in the Modern Era, it is rarely used to denote causality.

Similarly, as for conjunction, Kobayashi (1996) reports that to 'and' emerged in 17th Century. Also as can be seen in Figure 2, in the Mid-Edo period (Early 18th C) around $60 \%$ of the conjunction was expressed by $e-b a$, while $e$-ba is hardly used as conjunction in the Modern Era.

These markers that emerged later are semantically stronger than the default sequential conjunction. Let us first compare node and $e-b a$. The causal connective node is stronger than $e$-ba, i.e., there is a Q-scale, $\langle$ node, $e$-ba $\rangle$ since $\operatorname{CAUSE}(\varphi, \psi)$ entails $\varphi \rightarrow \psi$, but $\varphi \rightarrow \psi$ does not entail $\operatorname{CAUSE}(\varphi, \psi)$. Therefore, $\varphi-e-b a-\psi$ Q-implicates $\neg \operatorname{CAUSE}(\varphi, \psi)$. Similarly, the conjunction to is stronger than $e$-ba, i.e., $\langle$ to, $e-b a\rangle$, since $\varphi \& \psi$ entails $\varphi \rightarrow \psi$, but $\varphi \rightarrow \psi$ doesn't entail $\varphi \& \psi$. Thus, $\varphi$-e-ba- $\psi$ Q-implicates $\neg(\varphi \& \psi)$.

Figure 3 visualizes how the causal and conjunction interpretations of $e-b a$ in OJ are taken over by semantically stronger morphemes such as node and to. Until 14th 


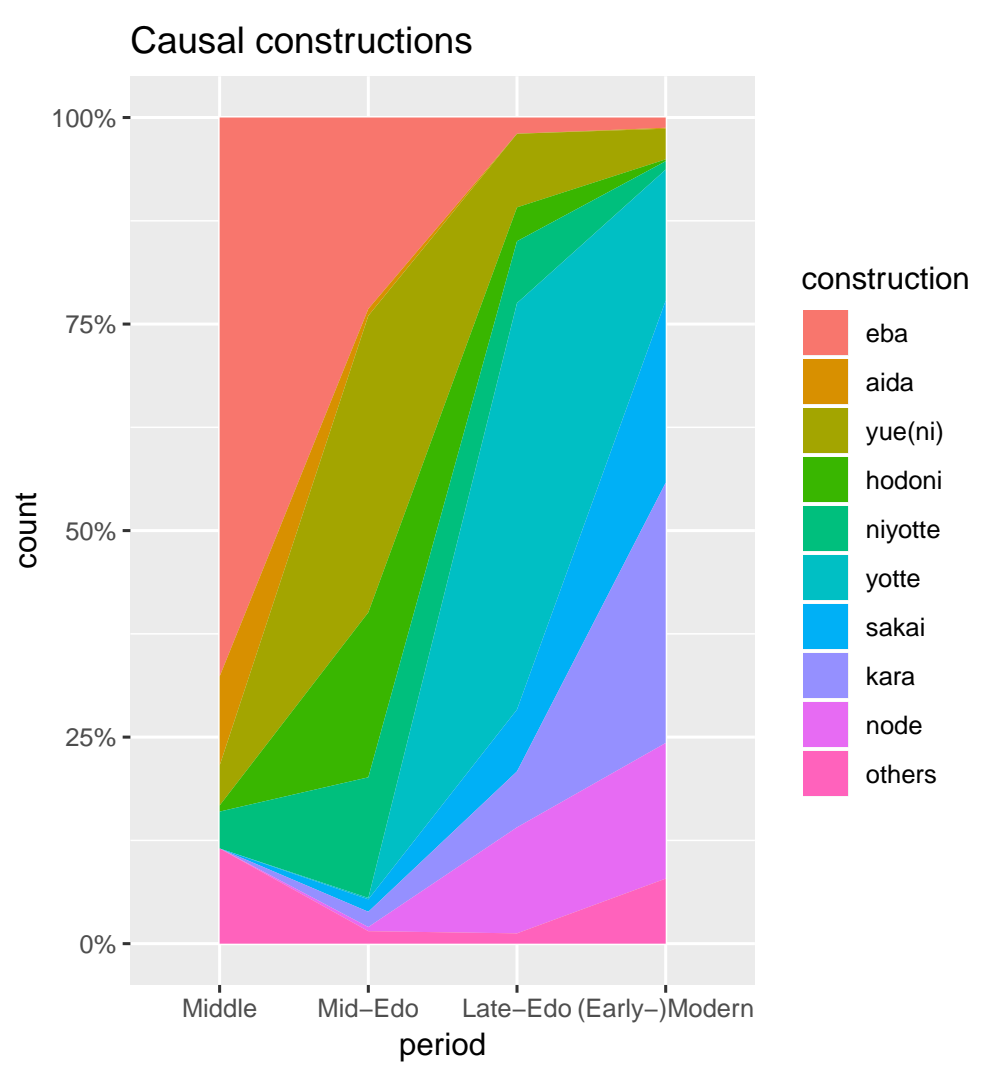

Figure 1 Constructions that mark causality (plotted based on Table 1 on page 217 in Yajima (2013))

Century when Heike Monogatari was written, $e$-ba was used mainly for causal or conjunction interpretations. After the causal node and the conjunction to entered the Japanese lexicon in 17th Century, the $e$-ba construction has acquired the conditional interpretation as its main use. Indeed, in the Mid-Edo Era (18th C), the causal/conjunction use of $e$ - $b a$ decreased while its conditional use reaches more than $50 \%$.

To recapitulate, in OJ, there was only a single construction V-e-ba to mark all three interpretations in question: sequential conjunction, logical/symmetric conjunction and causal. The OJ hearer had to use contextual information to disambiguate the OJ speaker's intended meaning for a successful communication.

Put another way, as summarized in (15), OJ was at the stage of zero-CAUS, where hearers had to use contextual information to disambiguate the meaning of $e$-ba. When the MidJ speakers started to use node 'because', Japanese entered the emergentCAUS stage. In ModJ, the interpretations of $e-b a$ and node are grammaticalized, thus 


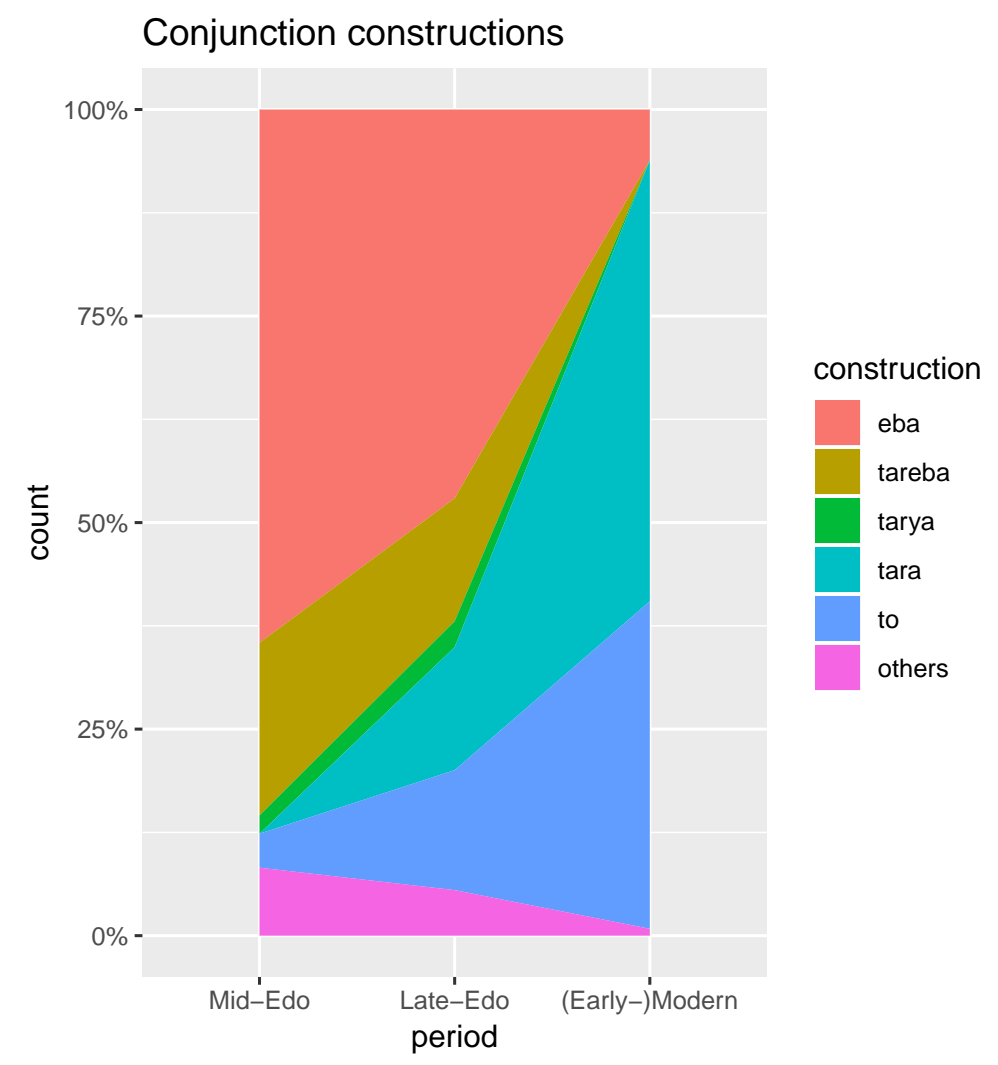

Figure 2 Constructions that mark conjunction (plotted based on Table 3 on page 113 in Yajima (2013))

ModJ is situated in the categorical-CAUS, where the speakers exclusively use $e-b a$ for conditionals and use node for causation.

(15) $\quad$ causal $\Rightarrow$ conditional

a. zero-CAUS: $e-b a$

b. emergent-CAUS: (node), $e-b a$

c. categorical-CAUS: node, $e-b a$

(ModJ)

Similarly, for the conjunction-to-conditional path (16), OJ was at the stage of zero-LCON (logical/symmetric conjunction). When the MidJ speakers started to use the lexicalized logical/symmetric conjunction to, it entered the emergent-LCON stage. In ModJ, the conditional interpretation of $e-b a$ and the conjunction interpretation of node are grammaticalized, thus the ModJ speakers use these morphemes categorically. 
Diachronic Semantic Shift of Sequential Conjunction

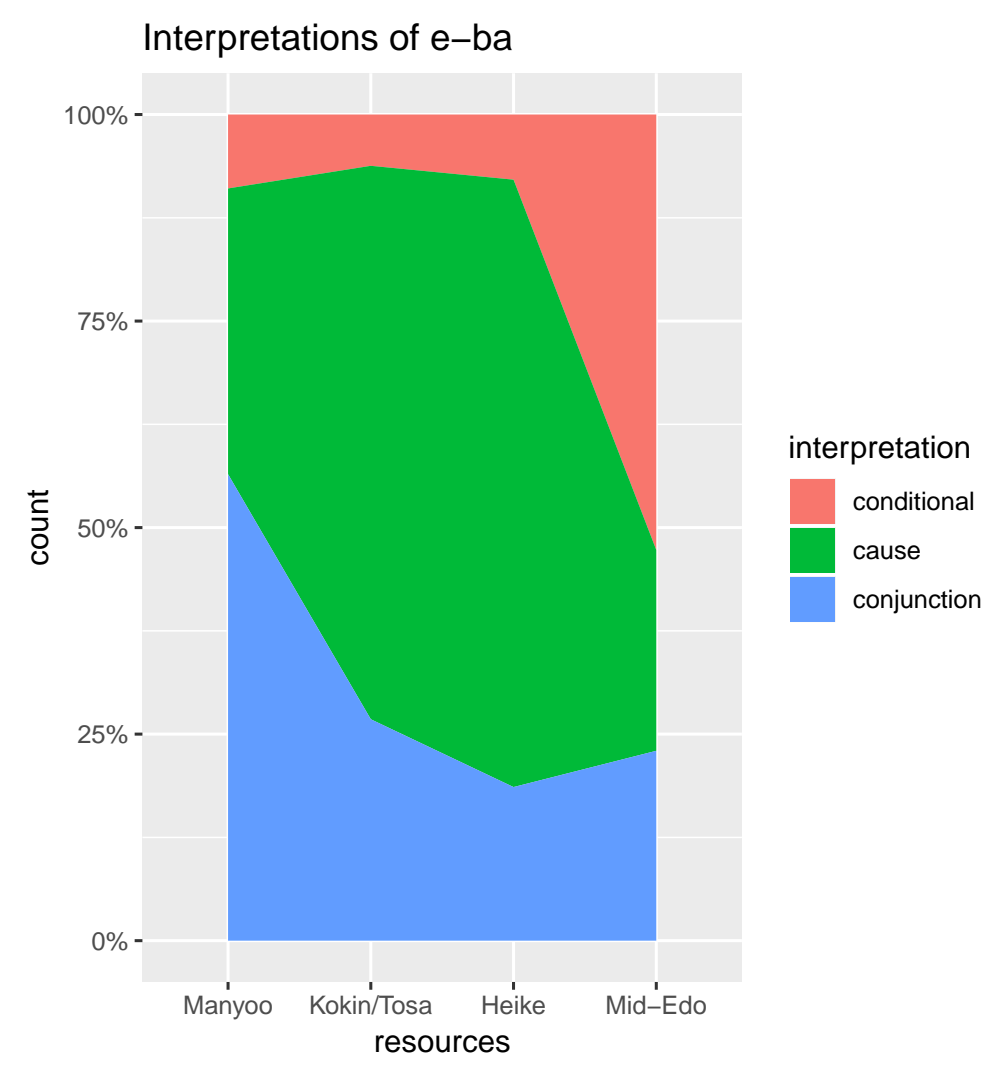

Figure 3 Interpretations of V-e-ba (plotted based on Tables $1 \& 2$ on pages $64 \& 66$ in Yajima (2013))

(16) conjunction $\Rightarrow$ conditional
a. zero-LCON: $e-b a$
(OJ)
b. emergent-LCON: (to), e-ba
(MidJ)
c. categorical-LCON: to, $e-b a$,
(ModJ)
d. generalized-LCON: to
(ModJ?)

Furthermore, as for the conjunction/conditional dichotomy, ModJ seems to be entering the generalized-LCON stage since $\varphi-t o-\psi$ has an interpretation similar to so-called "conditional conjunctions" (Culicover 1970; Kaufmann 2018) in (Modern) English as illustrated in (17).

(17) nonbiri siteru to okureru yo.

take.time PROG and late PRT

'You take time and you'll be late.'

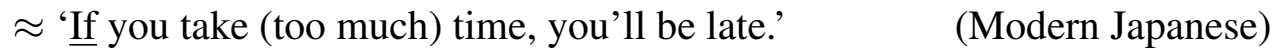


In short, both causal-to-conditional and conjunction-to-conditional paths follow the transition procedure in (18). At the end of the zero-CAUS and zero-LCON stages, some morphemes are recruited to specifically denote the stronger interpretations (Recruitment). Then, each morpheme is categorized to denote each interpretation (Categorization). Finally, at least as for the conjunction-to-conditional path, the newly recruited morpheme is partly generalized to have the conditional interpretation (Generalization).
a. Recruitment
b. Categorization
c. Generalization (conjunction $t o \rightarrow$ conditional to)

The next section models these grammaticalization transitions of the semantics of $e-b a$.

\section{EGT Modelling}

The diachrnoic trajectory sketched above naturally fits into the framework of Evolutionary Game Theory (van Rooij 2004; Deo 2015). In particular, Deo's (2015) analysis of the diacrhonic progressive-to-imperfective path is straightforwardly carried over to the current analyses of the causal-to-conditional and conjunction-toconditional paths. In the following, I mainly discuss the causal-to-conditional path for illustration. Also for reasons of space, the exposition of the model in the current paper is quite sketchy. Interested readers are referred to Deo (2015) and Yanovich $(2015) .^{2}$

\subsection{The E-ba Game}

We have two states/meanings, $T=\{$ caus(al), cond(itional) $\}$ and two signals/messages/linguistic forms, $M=\{$ node, $e-b a\}$. A speaker strategy $S$ is a function from states to forms while a hearer strategy $H$ is a function from forms to states. The $\delta$ function in (19) calculates the success of communication (Jäger 2007). The communication is successful when the hearer interprets $t$ from $S(t)$, the form chosen by the speaker.

$$
\delta_{t}(S, H)= \begin{cases}1, & \text { if } H(S(t))=t \\ 0, & \text { otherwise }\end{cases}
$$

We also factor in formal economy. Deo (2015) assumes that it is costly to have multiple signals for the same concept, which lessens the utility of a speaker's

2 I would like to thank Ashwini Deo (p.c.) for introducing Yanobich's (2015) work to me. 
Diachronic Semantic Shift of Sequential Conjunction

strategy. This assumption is implemented in (20). The parameter $k$ determines whether the speaker regards the success of communication with multiple forms or the cost of signal as more important (Jäger 2007). The function $n$ yields the number of expressions minus one.

(20) Speaker's Utility: $U_{s}(t, S, H)=\delta_{t}(S, H)-k \times n(S)$

(Deo 2015: 29)

Hearer's utility, on the other hand, is exactly the same as the $\delta$ function:

$$
\text { Hearer's Utility: } U_{h}(t, S, H)=\delta_{t}(S, H)
$$

(Deo 2015: 30)

Some states are more likely to be communicated than other states. Thus, the average utilities of speaker and hearer are measured by adding up the strategy utility in each state, which is loaded with probability weighting:
a. Speaker's average utility: $U_{S}(S, H)=\sum_{t} P(t) \times\left(\delta_{t}(S, H)-k \times n(S)\right)$
b. Hearer's average utility: $U_{h}(S, H)=\sum_{t} P(t) \times \delta_{t}(S, H)$
(Deo 2015: 30)

\subsection{Role of contexts}

Deo (2015) adopts van Rooij's (2004) model of signalling games enriched with contextual factors. In the current analysis, two contexts (phenomenal and structural) are considered, following Deo's (2015) hypothesis that "[a] semantic grammaticalization path in the functional domain must be structurally underpinned by some privative contrast between a specific and a general meaning" (p. 47). As for our causal-to-conditional and conjunction-to-conditional paths, we can indeed identify such a privative contrast as summarized in (23).

(23) Two kinds of contexts:

a. phenomenal context: specific event tokens-Causal/Conjunction

b. structural context: general event types-Conditional

A causal statement like (1a) describes a phenomenal relation between specific event tokens, i.e., the event that only harsh things increased caused the event that Ko'oi got very much depressed. Similarly, a conjunction of two clauses like (9) describes a phenomenal relation between two specific events, i.e., the event that the old man looked at the bamboo shoot chronologically precedes the event that a little person was sitting inside. On the other hand, a conditional statement like (2a) describes a structural relation between general event types, most events where you mention things sweetly coincide with events where men will love you more. 


\begin{tabular}{|lllll|}
\hline & \multicolumn{2}{c}{$C_{\text {phen }}$} & \multicolumn{2}{c|}{$C_{\text {struc }}$} \\
& caus & cond & caus & cond \\
$S_{c d}$ & $e-b a$ & $e-b a$ & $e-b a$ & $e-b a$ \\
$S_{p c d}$ & $e-b a$ & $e-b a$ & node & $e-b a$ \\
$S_{e m}$ & node & $e-b a$ & node & $e-b a$ \\
$S_{c d^{\prime}}$ & node & node & node & node \\
\hline
\end{tabular}

Table 3 Speaker strategies

In the current system, a context is a probability distribution over the state/meaning space. We have two contexts, $C=\left\{C_{\text {phen }}, C_{\text {struc }}\right\}$. In a phenomenal context, it is more likely that the agents communicate a causal meaning $\left(P_{\text {phen }}\right.$ (caus $)=$ $0.9 \& P_{\text {phen }}($ cond $)=0.1$ ) while in a structural context, it is more likely that the agents communicate a conditional meaning $\left(P_{\text {struc }}(\right.$ caus $)=0.1 \& P_{\text {struc }}($ cond $\left.)=0.9\right)$.

Deo's speaker and hearer strategies considered for the progressive-to-imperfective path are directly applied to the causal-to-conditional path as done in Tables 3 and 4. A speaker strategy $(S: C \times T \rightarrow M)$ is now a mapping from pairs of a state and a context to forms $\{$ node, $e-b a\}$.

$S_{c d}$ is a "context dependent" strategy where the speaker employs the $e$-ba form invariably. $S_{p c d}$ is a "partially context dependent" strategy where the speaker uses node to convey the caus state only in $C_{\text {struc }}$, where the cond state is more probable (i.e., $S_{p c d}\left(C_{s t r u c}\right.$, caus $)=$ node). $S_{e m}$ is an "explicit marking" strategy, where the speaker employs node to mean caus and $e$-ba to mean cond independently of contexts. $S_{c d^{\prime}}$ is the same as $S_{c d}$ except that the speaker invariably uses node instead of $e-b a$.

On the other hand, a hearer strategy is now a mapping from pairs of a form and a context to states, $H: C \times M \rightarrow T$.

When the hearer employs $H_{c d}$, the hearer ignores the linguistic form uttered by the speaker and determines the meaning solely from the context. When $H_{p c d}$ is employed, the hearer interprets caus only when the speaker utters node in $C_{\text {struc }}$ (i.e., $H_{p c d}\left(C_{s t r u c}\right.$, node $)=$ caus $)$. When $H_{e m}$ is employed, the hearer ignores the context and directly interprets node as caus and $e-b a$ as cond . Unlike the speaker strategy, $H_{c d^{\prime}}$ is unnecessary since it is exactly the same as $H_{c d}$.

After taking contexts into consideration, the average utilities of speaker and hearer are revised as follows:

$$
\begin{aligned}
& \text { a. } U_{s}(S, H)=\sum_{c} P(c) \times \sum_{t} P_{c}(t) \times\left(\delta_{t}(S, H)-k \times n(S)\right) \\
& \text { b. } U_{h}(S, H)=\sum_{c} P(c) \times \sum_{t} P_{c}(t) \times \delta_{t}(S, H)
\end{aligned}
$$


Diachronic Semantic Shift of Sequential Conjunction

\begin{tabular}{|lllll|}
\hline & \multicolumn{2}{c|}{$C_{p h e n}$} & \multicolumn{2}{c|}{$C_{s t r u c}$} \\
& node & $e-b a$ & node & $e-b a$ \\
$H_{c d}$ & caus & caus & cond & cond \\
$H_{p c d}$ & caus & caus & caus & cond \\
$H_{e m}$ & caus & cond & caus & cond \\
$H_{c d^{\prime}}$ & caus & caus & cond & cond \\
\hline
\end{tabular}

Table 4 Hearer Strategies

$\begin{array}{llll}\text { Strategies } & H_{c d} & H_{p c d} & H_{e m} \\ S_{c d} & 0.9 & 0.9 & 0.5 \\ S_{p c d} & 0.9-k & 0.95-k & 0.55-k \\ S_{e m} & 0.9-k & 0.95-k & 1-k \\ S_{c d^{\prime}} & 0.9 & 0.5 & 0.5\end{array}$

Table 5 Average utilities (taken from Deo 2015: 32)

Suppose now that $P\left(C_{\text {phen }}\right)=0.5$ and $P\left(C_{\text {struc }}\right)=0.5$ with the context values specified above. Table 5 summarizes the result of the calculation of the speaker's average utilities. The hearer's average utilities can be obtained by removing the parameter $k$.

When we communicate, we sometime play the role of speaker and sometime play the role of hearer. To make our model similar to the actual situation, we need to make the game symmetrized. A strategy is now defined as a pair, $\langle S, H\rangle$. The expected utility of one strategy with respect to another one is computed as in (25). Table 6 shows the result of the calculation of (25).

$$
\mathrm{EU}\left(\langle S, H\rangle,\left\langle S^{\prime}, H^{\prime}\right\rangle\right)=\frac{1}{2} \times\left(U_{S}\left(S, H^{\prime}\right)+U_{h}\left(S^{\prime}, H\right)\right)
$$

(Deo 2015: 33)

\subsection{Replication and Mutation}

To model the grammaticalization transitions summarized in (18), we now consider replication and mutation.

Suppose that $x_{A}$ is Strategy A's frequency (probability). The likelihood that one strategy is replicated by another individual is determined by the average payoff (fitness) of the strategy given in (26).
a. $f_{A}=a x_{A}+b x_{B}$
b. $f_{B}=c x_{A}+d x_{B}$

(Deo 2015: 36) 


$\begin{array}{ccccc}\text { Strategies } & \left\langle S_{c d}, H_{c d}\right\rangle & \left\langle S_{p c d}, H_{p c d}\right\rangle & \left\langle S_{e m}, H_{e m}\right\rangle & \left\langle S_{c d^{\prime}}, H_{c d}\right\rangle \\ \left\langle S_{c d}, H_{c d}\right\rangle & 0.9 & 0.9 & 0.7 & 0.9 \\ \left\langle S_{p c d}, H_{p c d}\right\rangle & 0.9-\frac{1}{2} k & 0.95-\frac{1}{2} k & 0.75-\frac{1}{2} k & 0.7-\frac{1}{2} k \\ \left\langle S_{e m}, H_{e m}\right\rangle & 0.7-\frac{1}{2} k & 0.75-\frac{1}{2} k & 1-\frac{1}{2} k & 0.7-\frac{1}{2} k \\ \left\langle S_{c d^{\prime}}, H_{c d}\right\rangle & 0.9 & 0.7 & 0.7 & 0.9\end{array}$

Table 6 Expected payoffs for paired strategies (taken from Deo 2015: 34)

Given the average payoff (fitness) of a strategy relative to the fitness of the population, the probability of replication of Strategy A after one time step $\left(x_{A}^{\prime}\right)$ is computed as in (27). $\phi$ is the average fitness of the population before the time step, $\phi=x_{A} f_{A}+x_{B} f_{B}$.

$$
\begin{aligned}
& \text { a. } x_{A}^{\prime}=x_{A} \times \frac{f_{A}}{\phi} \\
& \text { b. } x_{B}^{\prime}=x_{B} \times \frac{f_{B}}{\phi}
\end{aligned}
$$

(Deo 2015: 36)

Besides replication, Deo (2015) introduces mutation into the model. The motivation for introducing mutation from one strategy to another is to characterize the case that offsprings might learn a strategy different from the strategy of their parents. This paper directly adopts Deo's (2015) discrete-time version of the "replicator-mutator" equation in (28). The frequency of strategy $i$ after a time-step $\left(x_{i}^{\prime}\right)$ is determined on the basis of the probability that strategy $j$ mutates into strategy $i\left(Q_{j i}\right)$, the frequency of strategy $j\left(x_{j}\right)$, the average payoff of strategy $j\left(f_{j}\right)$ and the average fitness of the population $(\phi)$.

$$
x_{i}^{\prime}=\sum_{j=1}^{n} Q_{j i} \frac{x_{j} f_{j}}{\phi}
$$

(Deo 2015: 37)

\subsection{Modeling the transitions}

Finally, we are ready to model the grammaticalization transitions presented in (18). Let us take the replicator-mutator equation (28) and the mutation probabilities given in Table 7, and apply it to the causal-to-conditional and conjunction-to-conditional paths. The parameter $k$ is set to be 0.01 .

Recruitment Transition In the zero-CAUS stage, $\left\langle S_{c d}, H_{c d}\right\rangle$ is most common and easy to learn, although some learners may move to $\left\langle S_{p c d}, H_{p c d}\right\rangle$ to avoid ambiguity and miscommunication. According to Table 7 , the mutation probability from 
Diachronic Semantic Shift of Sequential Conjunction

$Q=\begin{array}{ccccc} & \left\langle S_{c d}, H_{c d}\right\rangle & \left\langle S_{p c d}, H_{p c d}\right\rangle & \left\langle S_{e m}, H_{e m}\right\rangle & \left\langle S_{c d^{\prime}}, H_{c d}\right\rangle \\ \left\langle S_{c d}, H_{c d}\right\rangle & 0.94 & 0.06 & 0 & 0 \\ \left\langle S_{p c d}, H_{p c d}\right\rangle & 0.02 & 0.91 & 0.07 & 0 \\ \left\langle S_{e m}, H_{e m}\right\rangle & 0 & 0 & 0.97 & 0.03 \\ \left\langle S_{c d^{\prime}}, H_{c d}\right\rangle & 0 & 0 & 0 & 1\end{array}$

Table 7 Mutation Probability given by Deo (2015: 41)

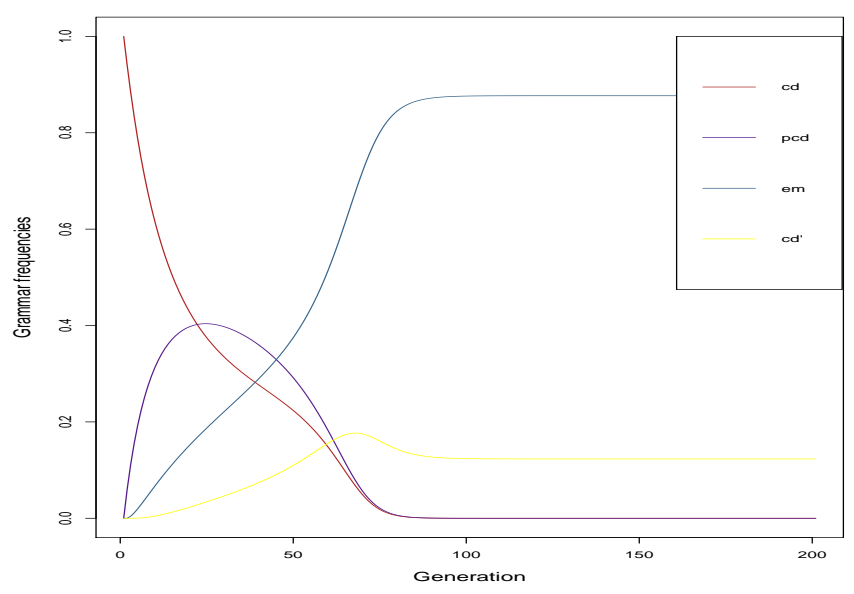

Figure 4 Dynamic behaviors of the four strategies based on $Q$ (replica of Figure 3 in Deo (2015: 43); R codes provided by Yanovich (2015))

$\left\langle S_{c d}, H_{c d}\right\rangle$ to $\left\langle S_{p c d}, H_{p c d}\right\rangle$ is 0.06 , i.e., $Q_{c d, p c d}=0.06$. The simulation based on the replicator-mutator equation (28) and the mutation probability matrix $Q$ in Table 7 is given in Figure 4. As can be seen, $\left\langle S_{p c d}, H_{p c d}\right\rangle$ wins over $\left\langle S_{c d}, H_{c d}\right\rangle$ after about 20 iterations.

Categorization Transition $\left\langle S_{p c d}, H_{p c d}\right\rangle$ prevalent in emergent-CAUS is a demanding strategy since the speaker needs to be attentive to the context, thus offsprings tend to go for $\left\langle S_{e m}, H_{e m}\right\rangle$ with the probability $Q_{p c d, e m}=0.07$ since the parent strategy contains node, an indication toward the grammaticalization of CAUS. $\left\langle S_{e m}, H_{e m}\right\rangle$ common in categorical-CAUS is a reliable strategy as can be seen in the simulation (Figure 4).

Generalization Transition Finally, to understand the generalization of the conjunction to to conditional to as seen in (17), let us see how Deo (2015) models the 


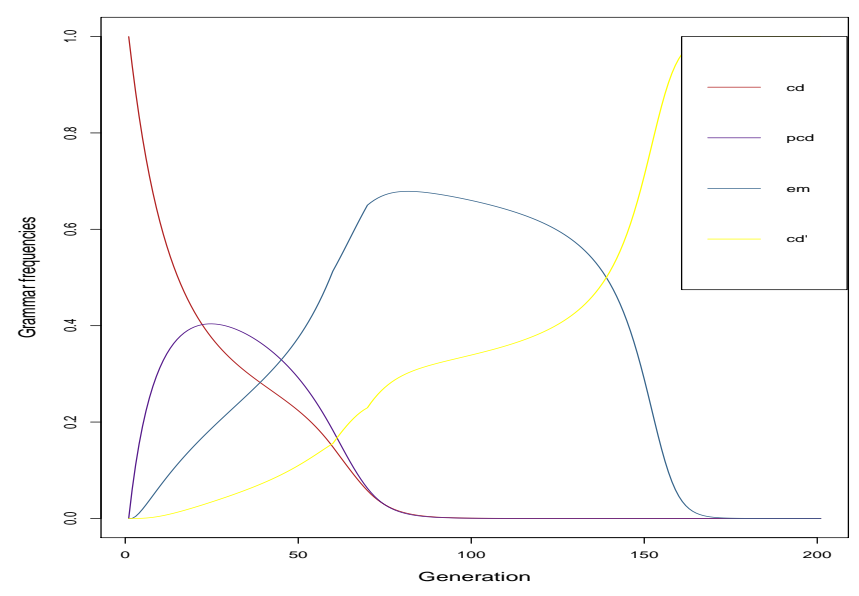

Figure $5\left\langle S_{e m}, H_{e m}\right\rangle$ to $\left\langle S_{c d^{\prime}}, H_{c d}\right\rangle$ with manipulated mutations (replica of Figure 4 in Deo (2015: 45); R codes provided by Yanovich (2015))

transition from $\left\langle S_{e m}, H_{e m}\right\rangle$ to $\left\langle S_{c d^{\prime}}, H_{c d}\right\rangle$. Basically, the mutation probability from $\left\langle S_{e m}, H_{e m}\right\rangle$ to $\left\langle S_{c d^{\prime}}, H_{c d}\right\rangle$, i.e., $Q_{e m, c d^{\prime}}$, is manipulated. The motivation behind this manipulation is the following: The increase in the frequency of to in the offspring input may lead to an increase of the chance in mis-learning a different strategy. Together with the assumption that learners prefer a simple grammar with a single form over another grammar with multiple forms, it is possible that as more agents employ the $\left\langle S_{e m}, H_{e m}\right\rangle$ strategy, there is an increase in the probability of the mutation into the $\left\langle S_{c d^{\prime}}, H_{c d}\right\rangle$ strategy.

To see how this manipulation results in the desired transition, let us see how Deo (2015) manipulates $Q_{e m, c d^{\prime}}$ with concrete numbers. When the frequency of $\left\langle S_{e m}, H_{e m}\right\rangle\left(x_{\left\langle S_{e m}, H_{e m}\right\rangle}\right)$ hits $0.5, Q_{e m, c d^{\prime}}$ rises from 0.03 to 0.04 . When $x_{\left\langle S_{e m}, H_{e m}\right\rangle}$ arrives at $0.65, Q_{e m, c d^{\prime}}$ further rises to 0.05 . The simulation with this manipulation is depicted in Figure 5.

\section{Conclusion}

\subsection{Summary}

This paper analyzed the diacrhonic semantic shift of the V-e-ba construction in Japanese. I propose that the conventional meaning or semantic denotation of the construction is a sequential conjunction, i.e., $c[\varphi][\psi]$, which can derive all the interpretations, i.e., causal, logical/symmetric conjunction and conditional, found throughout the history of Japanese. In particular, the causal interpretation in OJ is 
Diachronic Semantic Shift of Sequential Conjunction

obtained as an I-implicature, while the conditional interpretation in ModJ is obtained via Q-implicatures.

Furthermore, the grammaticalization transitions involved in the diachronic semantic shift of the V-e-ba construction, i.e., recruitment, categorization and generalization, are modeled within the framework of Deo's (2015) Evolutionary Game Theory.

\subsection{Remaining questions}

There are a lot of remaining questions in the analysis presented in the paper. First of all, what happened to the $a$ - $b a$ conditional? Why did it disappear? Some other conditional constructions in Japanese are reminiscent of the $a$ - $b a$ conditional. For instance, conditional markers tara and nara appear to be derived from tar-a-ba (PERFECT-A-BA) and nar- $a$ - $b a$ (COPULA-A-BA) by dropping the final $b a$, respectively. However, this line of analysis is not uncontroversial. Matsushita (1928) provides a quite convincing argument that tara and nara are derived from tarya and narya by dropping the palatalization, which in turn are contracted forms of tar-e-ba and nar-e-ba.

Secondly, these other forms of conditionals available in Japanese should certainly interact with the diachronic development of $e-b a$.

Finally, what is the particle $b a$ in $e-b a$ and $a-b a$ ? Fukuda (2006) claims that it is ambiguous between conjunction and conditional. However, there is also a convincing analysis by Ohno (1982), who argues that the particle $b a$ is etymologically related to the topic marker wa, which used to be pronounced as [pa] in OJ. According to Ohno (1982), V-a-ba is derived from V-a-m-pa (V-A-MODAL-TOPIC), where [p] phonologically changed into $[\mathrm{b}]$ as a result of sequential voicing.

\section{References}

Culicover, Peter. 1970. One more can of beer. Linguistic Inquiry 1. 366-369. https://www.jstor.org/stable/4177578.

Deo, Ashwini. 2015. The semantic and pragmatic underpinnings of grammaticalization paths: The progressive to imperfective shift. Semantics and Pragmatics 8(14). 1-52. doi:10.3765/sp.8.14.

Fukuda, Yoshiichiro. 2006. Jooken hyoogen no han'i: Koten nihongo no setsuzoku joshi ba o megutte. In Jooken hyoogen no taishoo, Kurosio.

Heim, Irene. 1982. The semantics of definite and indefinite noun phrases: University of Massachussets, Amherst PhD dissertation. [Distributed by GLSA].

Huang, Yan. 2007. Pragmatics Oxford Textbooks in Linguistics. New York: Oxford University Press. 
Jäger, Gerhard. 2007. Evolutionary game theory and typology: A case study. Language 83(1). 74-109. doi:10.1353/lan.2007.0020. http://www.jstor.org/ stable/4490338.

Kaufmann, Magdalena. 2018. Topics in conditional conjunctions. Slides presented at the 49th Annual Meeting of the North East Linguistic Society (NELS 49).

Kaufmann, Stefan. 2000. Dynamic context management. In S. Kaufmann M. Faller \& M. Pauly (eds.), Formalizing the dynamics of information, Stanford, CA: CSLI.

Kobayashi, Kenji. 1996. Nihongo jooken hyoogenshi no kennkyuu. Hitsuji Syobo.

Kratzer, Angelika. 1991. Conditionals. In A. von Stechow \& D. Wunderlich (eds.), Semantics: an international handbook of contemporary research, 651-656. Berlin: De Gruyter.

Levinson, Steven C. 2001. Presumptive meanings. Cambridge, Massachusetts: The MIT Press.

Matsushita, Daizaburoo. 1928. Kaisen hyoojun nihongo bunpoo [revised standard japanese grammar]. Kigensha.

Ohno, Susumu. 1982. Kanazukai to joodaigo [Kana writing and acient Japanese]. Iwanami Shoten.

Roberts, Craige. 1996. Information structure in discourse: Towards an integrated formal theory of pragmatics. In Jae Hak Yoon \& Andreas Kathol (eds.), Ohio state university working papers in linguistics, vol. 49, 91-136. The Ohio State University.

van Rooij, Robert. 2004. Signalling games select Horn strategies. Linguistics and Philosophy 27(4). 493-527. doi:10.1023/B:LING.0000024403.88733.3f.

Sakakura, Atsuyoshi. 1958. Jooken hyoogen no nagare. Kokugogaku 33.

Stalnaker, Robert. 1968. A theory of conditionals. In N. Resher (ed.), Studies in logical theory, Oxford: Blackwell.

Sweetser, Eve. 1990. From etymology to pragmatics: Metaphorical and cultural aspects of semantic structure. Cambridge: Cambridge University Press.

Traugott, Elizabeth Closs \& Richard B. Dasher. 2002. Regularity in semantic change. Cambridge; New York: Cambridge University Press.

Yajima, Masahiro. 2013. Kamigata/Osakago ni okeru jooken hyoogen no shiteki tenkai (historical development of conditional expressions in Kamigata/Osaka langauge). Kasama Shoin.

Yanovich, Igor. 2015. Analyzing imperfective games. Tech. rep. DFG Center for Advanced Studies, University of Tübingen. https://semanticsarchive.net/Archive/ jAxYjUzY/. 
Diachronic Semantic Shift of Sequential Conjunction

Yurie Hara

Research Faculty of Media and Communication Hokkaido University,

Kita 17 Jou, Nishi 8 chome, Kitaku, Sapporo

Hokkaido 060-0817,

Japan

hara@imc.hokudai.ac.jp 